\title{
Review: psychoeducational interventions reduce depressive symptoms in cancer
}

\author{
Barsevick AM, Sweeney C, Haney E, et al. A systematic qualitative analysis of psychoeducational interventions for \\ depression in patients with cancer. Oncol Nurs Forum 2002 Jan-Feb;29:73-84.
}

\section{QUESTION: In patients with cancer, are psychoeducational interventions effective for reducing depressive symptoms?}

\section{Data sources}

Studies were identified by searching CINAHL, Medline, PsycLIT, and CancerLit (all from 1980-2000), and by reviewing bibliographies of relevant articles.

\section{Study selection}

Scientific studies or systematic reviews of scientific studies (ie, qualitative or quantitative reviews that examined specific hypotheses, used a comprehensive search strategy, and stated explicit conclusions) were selected if they compared a psychoeducational intervention with usual care or an attentional control group, and some level of depression was the measured outcome; practice guidelines based on research were also included. Exclusion criteria included studies of children with cancer, spouses of patients with cancer, and interventions that were not strictly psychoeducational (eg, exercise).

\section{Data extraction}

Data were extracted on study setting, sample size, demographic characteristics, diagnosis, key components of the intervention, study quality, and outcomes. The main outcome was depression (ie, the entire range of depressive symptoms, including normal sadness in response to loss, as well as chronic depressed emotional affect and clinical depression meeting criteria for psychiatric disorder) measured by a separate scale or as part of a composite measure.

\section{Main results}

36 randomised controlled trials (RCTs), 7 quasiexperimental studies, 5 descriptive studies, 3 quantitative systematic reviews (meta-analyses), 3 qualitative systematic reviews, and 1 practice guideline met the selection criteria. The results favoured psychoeducational interventions for depression in patients with cancer in 22 of 36 RCTs, the 3 qualitative systematic reviews, 2 of 3 quantitative systematic reviews, and 1 practice guideline. Experimental interventions included behavioural therapy, non-directive counselling, education interventions, combinations of $\geq 2$ interventions, and combinations of counselling or behavioural intervention with cancer education. Results are summarised in the table.

\section{Conclusion}

In patients with cancer, psychoeducational interventions are effective for reducing a range of depressive feelings and symptoms.
Source of funding:

Ortho Biotech

Products.

For correspondence: Ms A M Barsevick, Fox Chase Cancer Center, Philadelphia, PA, USA AM_Barsevick@fccc.edu

Psychoeducational interventions $v$ usual care or an attentional control for depressive symptoms in cancer

\begin{tabular}{lcc} 
Type of intervention & $\begin{array}{l}\text { Number of } \\
\text { studies }\end{array}$ & $\begin{array}{l}\text { Overall effect: number of } \\
\text { studies showing improvement } \\
\text { or no difference }\end{array}$ \\
\hline Behaviour therapy only & 17 & 11 improvement; 6 no difference \\
\hline Counselling only & 10 & 7 improvement; 3 no difference \\
\hline Education only & 7 & 4 improvement; 3 no difference \\
\hline Counselling and education & 8 & 6 improvement; 2 no difference \\
\hline Behaviour therapy and education & 4 & 1 improvement; 3 no difference \\
\hline
\end{tabular}

\section{COMMENTARY}

The systematic review by Barsevick et al addresses an important clinical question as to whether psychoeducational interventions can reduce the risk and incidence of depression in patients with cancer. 22 RCTs supported the conclusion that psychoeducational interventions benefit depressive symptoms. Recognising and treating depression in patients with cancer is clinically important. However, the lack of distinction between depressive symptoms and depressive syndromes make the results of this analysis difficult to generalise.

Clinical depression is common among patients with cancer, and is a risk factor for medical morbidity, mortality, and impaired quality of life. ${ }^{1}$ Despite abundant evidence supporting this association, scant empirical evidence exists on interventions to successfully treat depression in this population. ${ }^{1}$ A major factor affecting the evidence to date appears to be the lack of clarity regarding the effect of specific treatments on a wide range of depressive phenomena from mild depressive symptoms to major depressive illnesses.

Several factors may impede the recognition and treatment of depression in oncology settings. These include attribution of depression to poorly controlled or advancing cancer, confusion regarding the overlap of depressive symptoms and those associated with cancer illness and treatment, lack of understanding of the point at which depressive symptoms reach clinical significance, and the stigma that accompanies the diagnosis and treatment of psychiatric disorders. Although the review addresses an important clinical problem, the lack of a clear definition of depression as well as the variety of treatments assessed may limit our ability to apply these results in practice. Further research targeting the effectiveness of specific interventions for clearly defined depressive symptoms and syndromes is therefore needed to enable nurses to successfully intervene or provide referrals for the growing number of depressed cancer patients they encounter in clinical practice.

Jeannie V Pasacreta, RN, CS, PhD Associate Professor Yale University School of Nursing New Haven, Connecticut, USA

1 Pasacreta JV. Depressive phenomena, physical symptom distress, and functional status among women with breast cancer. Nurs Res 1997;46:214-21. 